\title{
Detection of auditory signals occurring at random times: Intensity and duration
}

\author{
DAVID M. GREEN and ALBERT F. SMITH \\ Laboratory of Psychophysics, Harvard University, Cambridge, Massachusetts 02138
}

\begin{abstract}
Observers attempted to detect a weak auditory signal presented in noise. The onset of the signal was determined by a Poisson process, and only responses occurring $1 \mathrm{sec}$ after signal onset were considered detections. Three latency distributions were measured, the time to a signal onset, the reaction time distribution, and the false-alarm distribution (of responses occurring before a signal onset). A simple two-state model is proposed to account for a discrepancy between the distribution of signal waits and the distribution of false alarms. The hazard functions of the reaction time distributions are considered in detail and a simple accumulation model is proposed to account for the results.
\end{abstract}

This research follows some previous efforts to analyze the detection of auditory signals presented at random times (Green \& Luce, 1967, 1971; Luce \& Green, 1970). It differs, therefore, from the majority of conventional experiments in psychophysics which employ a fixed observation interval. The motive for such a study is simply to provide a more rigorous analysis of the most common detection situation, for only rarely, outside the laboratory, do we detect signals that occur within well-marked temporal intervals. In our experiments, the signal onset is determined by a Poisson process. Thus, at any instant, there is a fixed and constant small probability that the signal will be presented. The observer is instructed to respond as soon as the signal is detected. If the observer responds before the signal is presented, we say that a false alarm has occurred and impose some penalty points. If the signal is not detected within $1 \mathrm{sec}$ of its onset, we say that a miss has occurred and impose some penalty points. If the observer responds within $1 \mathrm{sec}$ of the signal onset, we say that a hit has occurred and award a positive number of points. A trial, therefore, is terminated by a false alarm if no signal was presented or by a hit or a miss, depending on whether a response occurred within $1 \mathrm{sec}$ of the signal onset. A new trial begins approximately $1 \mathrm{sec}$ after the previous one has ended.

Two experimental variables were studied; they are signal duration and intensity. Signal intensity was studied because it is clearly an important parameter of such detection tasks. We need to know the range of intensity that one can use and still obtain reasonable estimates of hit and false-alarm probabilities. Duration is important because the behavior obtained

This research was supported in part by the National Science Foundation. We would like to thank G. B. Henning and R. D. Luce for reading and commenting on an earlier version of this paper. in these tasks may differ, depending on whether a long or short signal is employed.

Two series of experiments are reported. In the first, one duration was selected and intensity was varied over blocks of trials. Once the block was complete, a different intensity was selected. Not surprisingly, the criteria of the observers varied over the different intensities, as evident in variation of the falsealarm rate with intensity. Therefore, in the second series of experiments, one of three intensities was selected at random on each trial within a block, so that the observer was forced to use the same criterion over all intensities. In this way, the effect of intensity can be measured for a fixed criterion. For both experiments, the analysis of the data is the same.

The basic data consist of two distributions of response latencies. The false-alarm distribution is composed of times measured from the beginning of a trial to the time a response occurred, given that no signal had been presented. The second distribution, a reaction-time distribution, consists of times from the onset of the signal to when a response occurred, given that it occurred within $1 \mathrm{sec}$ of the signal onset. A third distribution of interest is the signal-wait distribution; signal waits are measured from the beginning of the trial to the onset of the signal. This distribution is determined jointly by the Poisson parameter, selected by the experimenter, which determines the probability of a signal onset, and by the observer's false-alarm rate. One may think of these two processes, signal onset and false alarm, as in a race to see which will occur first.

For all these distributions, we estimated the obvious parameters, mean and standard deviation. In addition, we calculated an estimate of the hazard function, the conditional probability of a response given that it had not yet occurred. The results will, we hope, illustrate why we believe the hazard functions to be important. 


\section{EXPERIMEN'TS}

\section{Procedure}

Three observers, in their late teens and early twenties, listening binaurally over TDH 50 earphones in sound-treated rooms, tried to detect a $1,000-\mathrm{Hz}$ sinusoidal signal added to a continuous background noise. The spectrum level of the noise was $35 \mathrm{~dB}$, and the signal intensity was varied at one of two signal durations, 50 and $1,000 \mathrm{msec}$. The observers were instructed to respond by pressing a button as soon as they thought they heard a signal.

Each trial began with a countdown of five $200-\mathrm{msec}$ events presented on a television terminal. Extinction of the final event and the appearance of the word "run" on the screen began the trial. A random wait $t \geqslant 0$, with density $\lambda e^{-\lambda t}$, where $\lambda=0.1 \mathrm{sec}$, was selected as the signal wait. Following any response or missed signal, a dead period of 1 sec occurred. During that period, the television screen identified the preceding event, showed the cumulative point total, and simultaneously began the five-event countdown. If a response occurred prior to signal onset, a "false alarm" was indicated; if the response occurred within $1 \mathrm{sec}$ of signal onset, the screen signaled a "hit." If no response occurred, the screen signaled a "miss." The points associated with a hit, a false alarm, and a miss were $100,-5$, and -10 , respectively. The cumulative points were known to the observers, but did not affect their pay in the experiment. The observers were paid $\$ 3.75 / \mathrm{h}$. Trials were run in blocks lasting about $12 \mathrm{~min}$. At the end of each block, the computer, a PDP-15, displayed the number of hits, false alarms, and misses, as well as the mean and standard deviation of the latencies associated with the false alarms and reaction times.

\section{Results}

Signal level varied over blocks. Two observers, D.S. and P.N., participated in this experiment in which three signal levels were selected and two different durations, 50 and $1,000 \mathrm{msec}$, were tested. Tables la and $1 \mathrm{~b}$ show the summary data for the two

Table 1

DS

(a) Summary of Brief (50-Msec) Signal-Level Varied Across Blocks

$\mathrm{E} / \mathrm{N}_{0}=15 \mathrm{~dB}$

1. Total Number of Responses

2. False Alarm Latency $\pm \sigma(\mathrm{N})$

3. False Alarm Tail $>1 \sec (N)$

4. Mean Reaction Time $\pm \sigma(\mathrm{N})$

5. Signal Wait Latency $\pm \sigma(\mathrm{N})$

6. Signal Wait Tail $>1 \sec (\mathrm{N})$

$\mathrm{E} / \mathrm{N}_{0}=12 \mathrm{~dB}$

1. Total Number of Responses

2. False Alarm Latency $\pm \sigma(\mathrm{N})$

3. False Alarm Tail $>1 \sec (\mathrm{N})$

4. Mean Reaction Time $\pm \sigma(\mathrm{N})$

5. Signal Wait Latency $\pm \sigma(N)$

6. Signal Wait Tail $>1 \sec (\mathrm{N})$

$\mathrm{E} / \mathrm{N}_{0}=9 \mathrm{~dB}$

1. Total Number of Responses

2. False Alarm Latency $\pm \sigma(\mathrm{N})$

3. False Alarm Tail $>1 \sec (\mathrm{N})$

4. Mean Reaction Time $\pm \sigma(\mathrm{N})$

5. Signal Wait Latency $\pm \sigma(N)$

6. Signal Wait Tail $>1 \sec (N)$

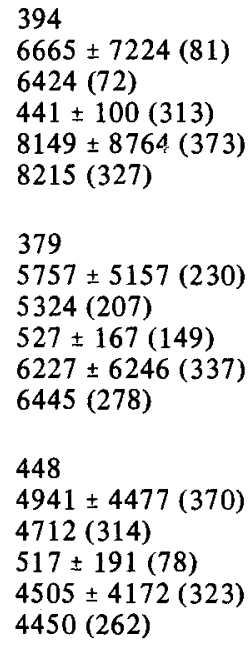

394

$6665 \pm 7224(81)$

$6424(72)$

$441 \pm 100(313)$

$8149 \pm 8764(373)$

8215 (327)

379

$5757 \pm 5157(230)$

5324 (207)

$527 \pm 167(149)$

$6227 \pm 6246(337)$

6445 (278)

448

$4941 \pm 4477(370)$

4712 (314)

$517 \pm 191(78)$

$4505 \pm 4172(323)$

4450 (262)

316

$7965 \pm 8238(74)$

$7951(65)$

$469 \pm 100(242)$

$8874 \pm 8666(344)$

$8860(308)$

290

$6772 \pm 6759(187)$

$6606(165)$

$463 \pm 134(103)$

$6626 \pm 7208(331)$

$6627(284)$

530

$5639 \pm 5669(205)$

$5358(179)$

$530 \pm 200(37)$

$6182 \pm 6079(356)$

$5998(311)$

$$
\begin{aligned}
& P(F A)=.177 \\
& P(D)=.703 \\
& P(F A)=.361 \\
& P(D)=.311 \\
& P(F A)=.365 \\
& P(D)=.104
\end{aligned}
$$

(b) Summary of Long (1,000-Msec) Signal-Level Varied Across Blocks

$\mathrm{E} / \mathrm{N}_{\mathrm{o}}=20 \mathrm{~dB}$

1. Total Number of Responses

2. False Alarm Latency $\pm \sigma(\mathrm{N})$

3. False Alarm Tail $>1 \sec (N)$

4. Mean Reaction Time $\pm \sigma(\mathrm{N})$

5. Signal Wait Latency $\pm \sigma(\mathrm{N})$

6. Signal Wait Tail $>1 \sec (\mathrm{N})$

$\mathrm{E} / \mathrm{N}_{0}=18 \mathrm{~dB}$

1. Total Number of Responses

2. False Alarm Latency $\pm \sigma(N)$

3. False Alarm Tail $>1 \sec (\mathrm{N})$

4. Mean Reaction Time $\pm \sigma(N)$

5 . Signal Wait Latency $\pm \sigma(N)$

6. Signal Wait Tail $>1 \sec (N)$

$\mathrm{E} / \mathrm{N}_{0}=16 \mathrm{~dB}$

1. Total Number of Responses

2. False Alarm Latency $\pm \sigma(\mathrm{N})$

3. False Alarm Tail $>1 \sec (\mathrm{N})$

4. Mean Reaction Time $\pm \sigma(\mathrm{N})$

5. Signal Wait Latency $\pm \sigma(N)$

6. Signal Wait Tail $>1 \sec (\mathrm{N})$

\section{5}

$8150 \pm 8185(54)$

$7599(51)$

$648 \pm 149$ (211)

$8213 \pm 7452(237)$

8091 (213)

427

$7091 \pm 7029(211)$

$6311(204)$

$647 \pm 203(216)$

$5548 \pm 5277(348)$

$5292(303)$

385

$6644 \pm 5926(253)$

$6007(239)$

$650 \pm 244$ (132)

$5527 \pm 6104(317)$

$5618(261)$
374

$8089 \pm 6366(106)$

$7372(102)$

$677 \pm 160(268)$

$8169 \pm 8937(332)$

$7992(300)$

519

$\mathrm{P}(\mathrm{FA})=.377 \quad 5556 \pm 4073(315)$

4820 (299)

$P(D)=.621$

$693 \pm 191(204)$

$4334 \pm 4051(357)$

4250 (289)

280

$\mathrm{P}(\mathrm{FA})=.444 \quad 5823 \pm 5133(230)$

5229 (213)

$P(D)=.416$

$616 \pm 243(50)$

$6404 \pm 7029(325)$

6358 (280)

$$
\begin{aligned}
& \mathrm{P}(\mathrm{FA})=.248 \\
& \mathrm{P}(\mathrm{D})=.807
\end{aligned}
$$

$\mathrm{P}(\mathrm{FA})=.469$

$\mathrm{P}(\mathrm{D})=.571$

$\mathrm{P}(\mathrm{FA})=.414$

$P(D)=.154$ 
signal durations. At each signal level, a number of summary statistics is given; their format is as follows. The first row is the total number of responses-this is simply the total number of times the observer pressed the button in this condition of the experiment. The mean latency, standard deviation, and number of false alarms are given in the second row. In a separate column, we also list the probability of a false alarm, $P(F A)$, the proportion of trials terminated by a false alarm. The third row gives the mean latency, minus $1 \mathrm{sec}$, of all false-alarm responses exceeding $1 \mathrm{sec}$; that is, we subtract $1 \mathrm{sec}$ from all falsealarm latencies, throw away any negative values, and compute the mean. We will call this a tail distributionit has some theoretical importance and will be used in the discussion section. The fourth row shows the mean latency, standard deviation, and number of responses that occurred within $1 \mathrm{sec}$ after the signal onset. Recall that if no response occurred within $1 \mathrm{sec}$ after signal onset, the trial terminated and a new trial began. In a separate column, we list the probability of a signal detection, that is, the number of responses within $1 \mathrm{sec}$ of the signal onset divided by the total number of signals presented. Row 5 is the mean latency, standard deviation, and number of signal waits, that is, the time between the trial onset and the onset of the signal. Row 6 lists the mean signal wait latency, minus $1 \mathrm{sec}$, of all signal waits exceeding $1 \mathrm{sec}$, that is, the mean of the signal-wait tail. The number of such events is listed after the mean.

Several features of the tabulated data deserve mention: (1) The probability of detection increases with signal level. The exact functional form of this relation is difficult to estimate because (2) the falsealarm rate diminishes with increasing signal level except for one reversal, Observer P.N. at the lowest signal level with the long-duration signal. The observers were asked to hold a constant criterion, but, at the highest signal level, a high false-alarm rate proved difficult to maintain and both observers had noticeably smaller false-alarm rates with both signal durations. (3) For both the false-alarm and signal-wait distributions, the mean of a given condition and standard deviation for that condition are nearly equal. This feature will be discussed in more detail in the theoretical section. (4) The mean reaction time is nearly constant as a function of signal level for the long-duration signal and changes only slightly and somewhat erratically with level at the shorter signal level. This relation is difficult to study, since the mean is probably influenced by the false-alarm probability, which changes with signal level.

Figures $1 \mathrm{a}$ and $1 \mathrm{~b}$ show backward cumulative distributions for both the signal-wait and false-alarm distributions for each signal duration at the medium signal level. For each distribution, we plot the natural logarithm of the probability of a response (or wait)

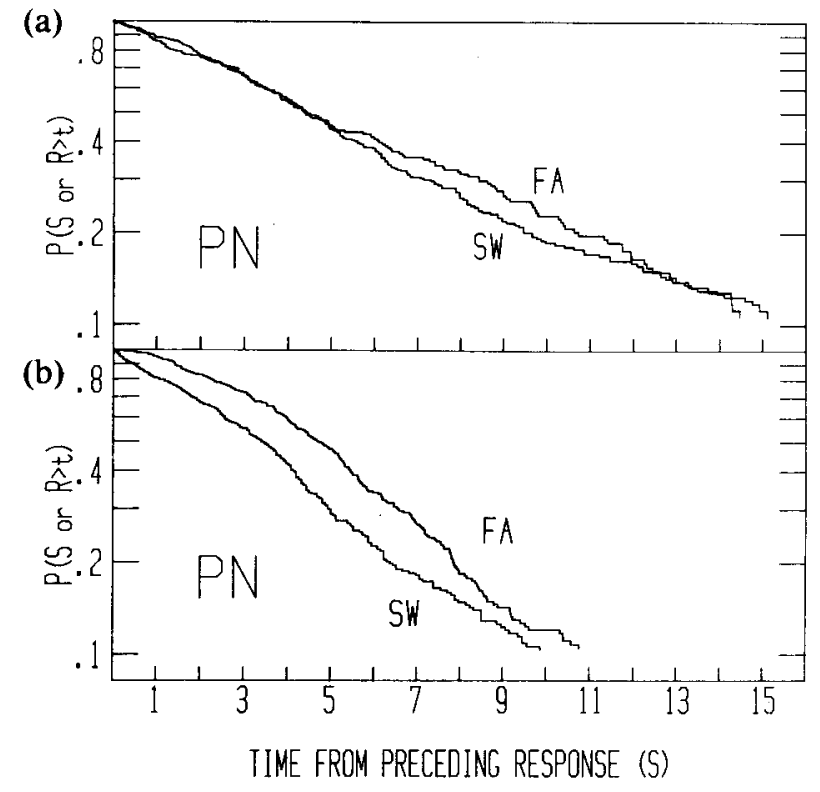

Figure 1. Backward cumulative plots of signal-wait and falsealarm distributions for the medium signal level, for Observer P.N. Panel a shows the data for the 50-msec signal duration; panel b shows the data for the 1,000 -msec signal duration.

exceeding the time indicated on the abscissa. Except for the short times of the false-alarm distributions, and for the very long times, for which there is a paucity of data in both distributions, the curves can be approximated by straight lines. This implies that the latency distributions are exponential in form.

Estimates of the reaction-time distributions are shown in Figures $2 a$ and $2 b$. These are constructed by counting the responses in consecutive $50-\mathrm{msec}$ bins, dividing by the total number of signals, and connecting these values with straight-line segments. These distributions are estimates of the probability of a particular reaction time, given that a signal occurred; the area under these curves is an estimate of the probability that a detection occurred, and thus varies with signal level in the expected manner. Of more interest are the hazard functions, Figures 3a and $3 \mathrm{~b}$. These are constructed from the reactiontime distributions by dividing the estimated relative frequency (number of responses per signal) in each $50-\mathrm{msec}$ bin by the complement of the relative frequency cumulated in all bins up to this bin. This value is then scaled so that an exponential wait with a mean of $1 \mathrm{sec}$ will yield a constant hazard function with a value of unity. The hazard functions for the long and short durations are distinctly different. For the long-duration signal, the probability of a response, given that one has not yet occurred, rises slowly and reaches a plateau after about 600 to $700 \mathrm{msec}$. The function is ragged at the longest times, because the number of events is small, but appears to remain 


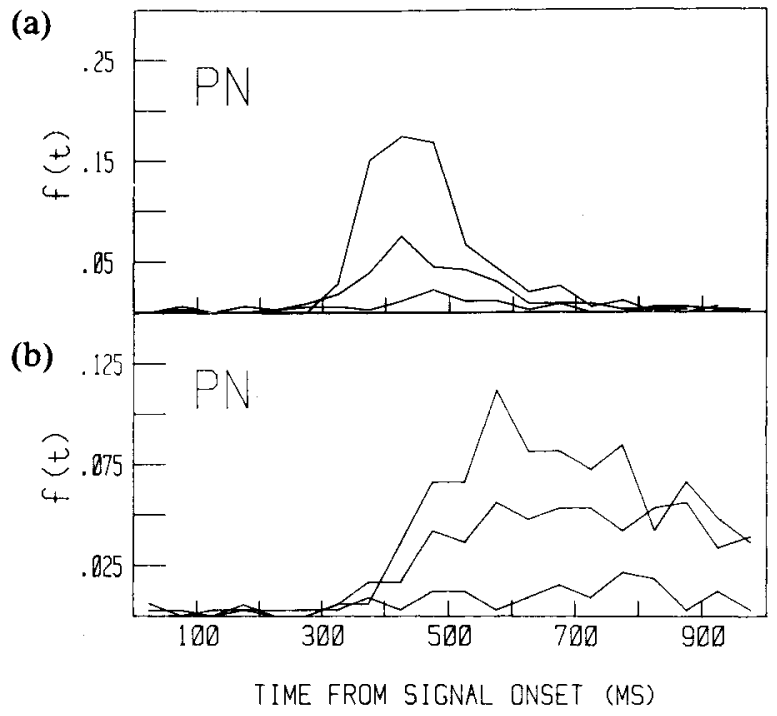

Figure 2. Reaction time distributions for Observer P.N. Panel a shows the data for the 50 -msec signal duration at three signal levels; panel $b$ is comparable data for the 1,000 -msec signal duration.

constant until the trial terminates. The hazard function for the short-duration signal is quite different. It appears to rise much sooner than does that for the long-duration signal, peaks at about 400 to $500 \mathrm{msec}$, and then diminishes to near zero by the end of the interval. The probability of detecting a brief signal after $\mathbf{8 0 0} \mathrm{msec}$, given that it has not been detected, is virtually nil.

For the longer duration signal, Figure $3 b$, there is little indication of any overshoot, that is, a peak higher than the asymptotic level. A change or transient detector would produce some initially high level of detection that diminishes as one moves away from the signal onset. Thus, no evidence of such a process is apparent. Both long- and short-duration hazard functions are consistent with a simple accumulation process having an effective integration time of about $300-400 \mathrm{msec}$, and a motor component of about 200$300 \mathrm{msec}$ that is statistically independent of the other detection processes. The absence of overshoot is not due to the rather gross $(50-\mathrm{msec})$ intervals used to compute the hazard function; analysis with finer (10-msec) intervals yields similar results.

Signal level randomized within blocks. Because observers varied their response criteria with changes in signal level, we undertook a more extensive series of measurements with the signal randomly selected from one of three possible levels on each trial. Another observer, D.T., was added to the experiment. For the short-duration signal, the same three signal levels were used. For the long-duration signal, we increased the ratio of successive signal levels to $3 \mathrm{~dB}$ and used values of $E / N_{0}=20,17$, and $14 \mathrm{~dB}$.

Tables $2 a$ and $2 b$ summarize the data in the style of Table 1. The columns marked P(FA) in the second row give the ratio of the number of trials that result in false alarms and the total number of trials. As can be seen, it ranges from about 0.4 to a little over 0.5 . The columns marked P(D) are estimates of the probability of detection, calculated by dividing the number of responses falling within $1 \mathrm{sec}$ of the signal onset by the total number of signals presented; this number increases systematically with signal intensity. The relatively large changes of the probability of detection with level in this experiment compared with those observed in the earlier experiment make it likely that changes in the earlier experiment were attenuated by variation in response criterion. Again, the mean is nearly the same as the standard deviation for both signal-wait and false-alarm distributions. For the false-alarm distribution, there are noticeable changes in the means if we exclude all responses occurring before $1 \mathrm{sec}$. This means that fewer responses occurred in the first second than one would expect in an exponential distribution. This reflects a tendency on the part of many observers not to realize that the most likely termination of an exponential wait is the first instant after zero. They seem to believe a signal will occur only at later times, despite our repeated remindings to the contrary. This is not characteristic of the stimulus-wait distribution.

The mean reaction time has a tendency to be shorter for the more intense signal, but this trend is reasonably clear only for the $50-\mathrm{msec}$ signal. For the longduration signal, only the most intense signal generates a clearly faster response.

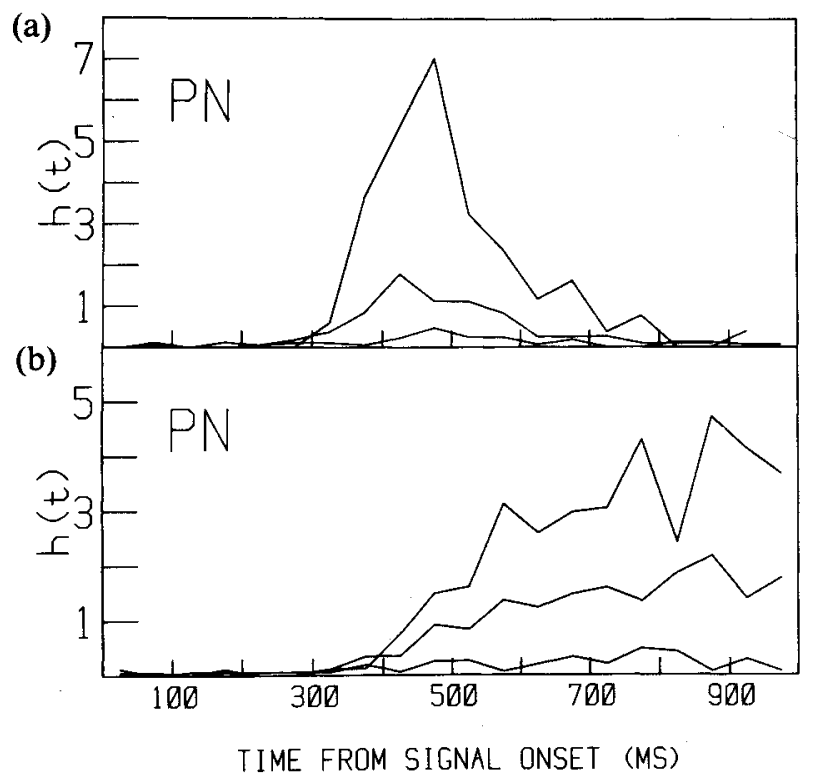

Figure 3. Hazard function based on the reaction time data of Figure 2. Panel a shows the data at the three signal levels for the 50-msec signal duration; panel b shows similar data for the 1,000msec signal duration. 
Table 2

DS

DT

PN

(a) Summary of Brief (50-Msec) Signal-Within-Block Data

1. Total Number of Responses 2984 1198

1817

$\mathrm{P}(\mathrm{FA})$

P(FA)

P(FA)

2. False Alarm Latency $\pm \sigma(N)$

$5027 \pm 4609(1849)$

3. False Alarm Tail $>1 \sec (\mathrm{N})$ 4775 (1585)

4. Mean Reaction Time $\pm \sigma(N)$ Signal Level 15

Signal Level 12

Signal Level 9

$416 \pm 102(598)$
$478 \pm 145(385)$
$516 \pm 188(151)$
$5166 \pm 5080(2002)$
$5137(1659)$
$4816(621)$

$\mathrm{P}(\mathrm{D})$

.930

.564

.224

$476 \pm 113(311)$

$520 \pm 146(156)$

$495 \pm 188$ (54)

$6496 \pm 7013(1030)$

$6525(880)$

.397

$6026 \pm 5992(1075)$

.397

$5523(984)$

P(D)

.876

.473

.156

$436 \pm 103(451)$

$471 \pm 146(215)$

$495 \pm 226(75)$

$6314 \pm 6579(1706)$

6294 (1461)

(b) Summary of Long (1,000-Msec) Signal-Within-Block Data

1. Total Number of Responses 3223

2. False Alarm Latency $\pm \sigma(\mathrm{N})$

3. False Alarm Tail $>1 \sec (\mathrm{N})$

$5634 \pm 5250(1869)$
$5164(1692)$
1694

P(FA)

.461

4606

$4606 \pm 4066(1144)$

P(FA)

3759 (1100)

$562 \pm 166(697)$

$663 \pm 184(454)$

Signal Level 20

Signal Level 17

Signal Level 14

5. Signal Wait Latency $\pm \sigma$ (N)

6. Signal Wait Tail $>1 \sec (N)$
$651 \pm 230(202)$

$4966 \pm 4903$ (2184)

4884 (1815)
P(D)

.948

.609

.287

$617 \pm 175(271)$

$641 \pm 222(181)$

$627 \pm 264(98)$

$4771 \pm 5323(977)$ 4821 (785)
3271

$4941 \pm 4386(2145)$

$\mathrm{P}(\mathrm{FA})$

.504

4258 (1996)

P(D)

.903

.531

.292

$619 \pm 173(597)$

$664 \pm 208(361)$

$654 \pm 220$ (167)

$\mathrm{P}(\mathrm{D})$

$4987 \pm 5559(2108)$

5084 (1696)

Note-Time is measured in milliseconds unless otherwise indicated.

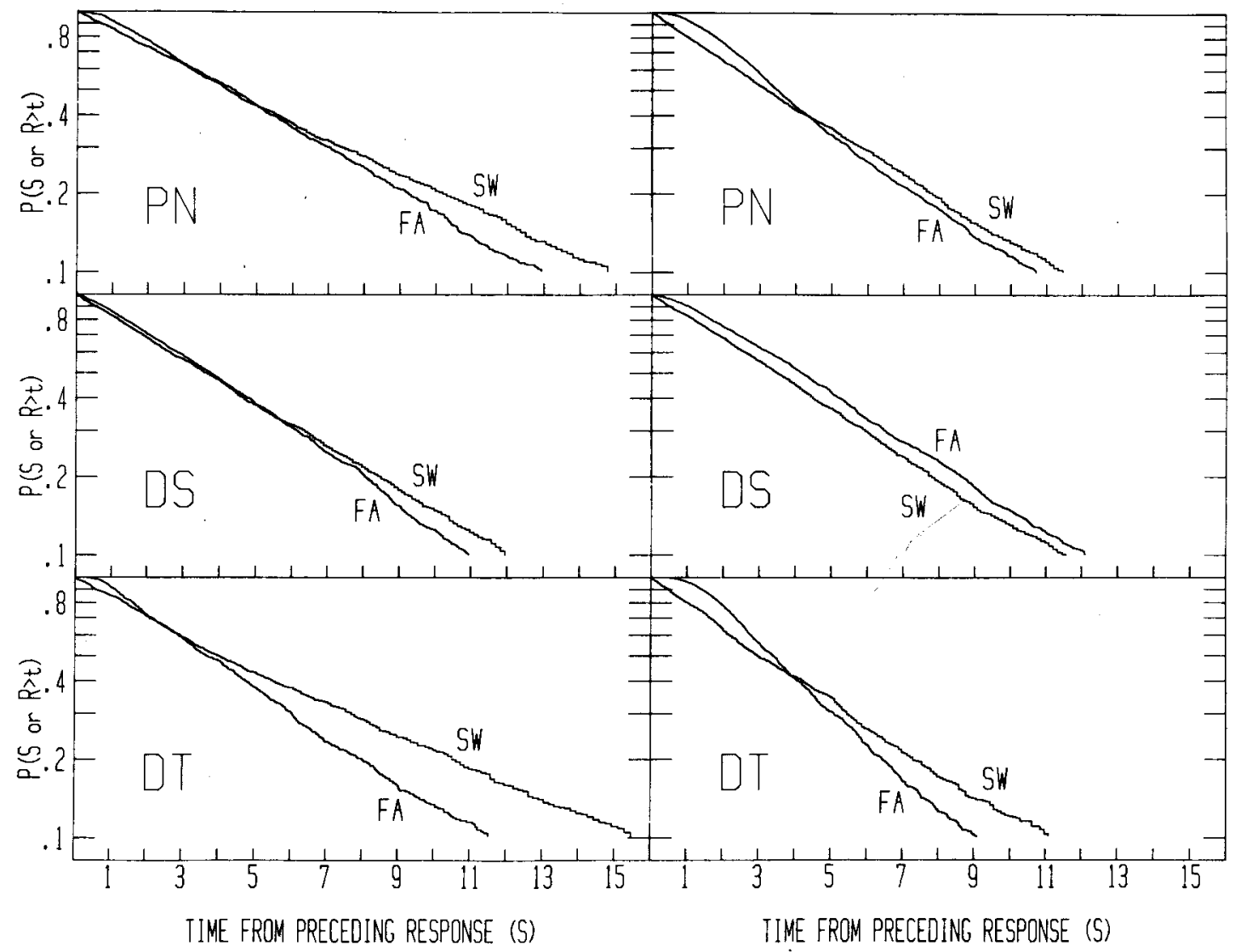

Figure 4. Backward cumulative plots of the signal-walt and false-alarm distributions for wh three observers. The left panel shows data for the 50-msec signal duration and the right panel, for the 1,000-msec signal duration. 

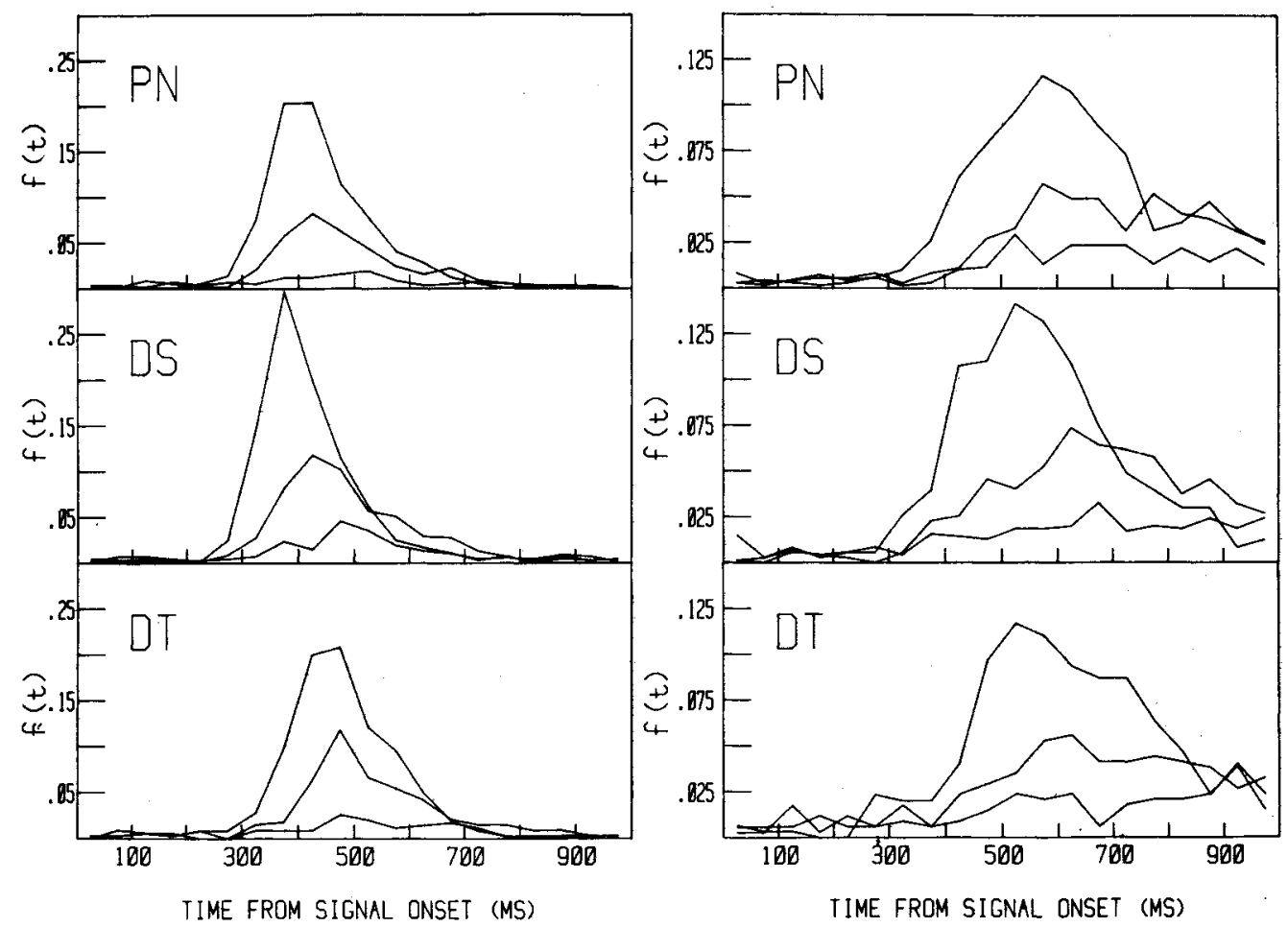

Figure 5. Reaction time distribution for three observers and the three signal levels used in that experiment. The left panel shows data for the 50-msec signal duration and the right panel, for the 1,000-msec signal duration.
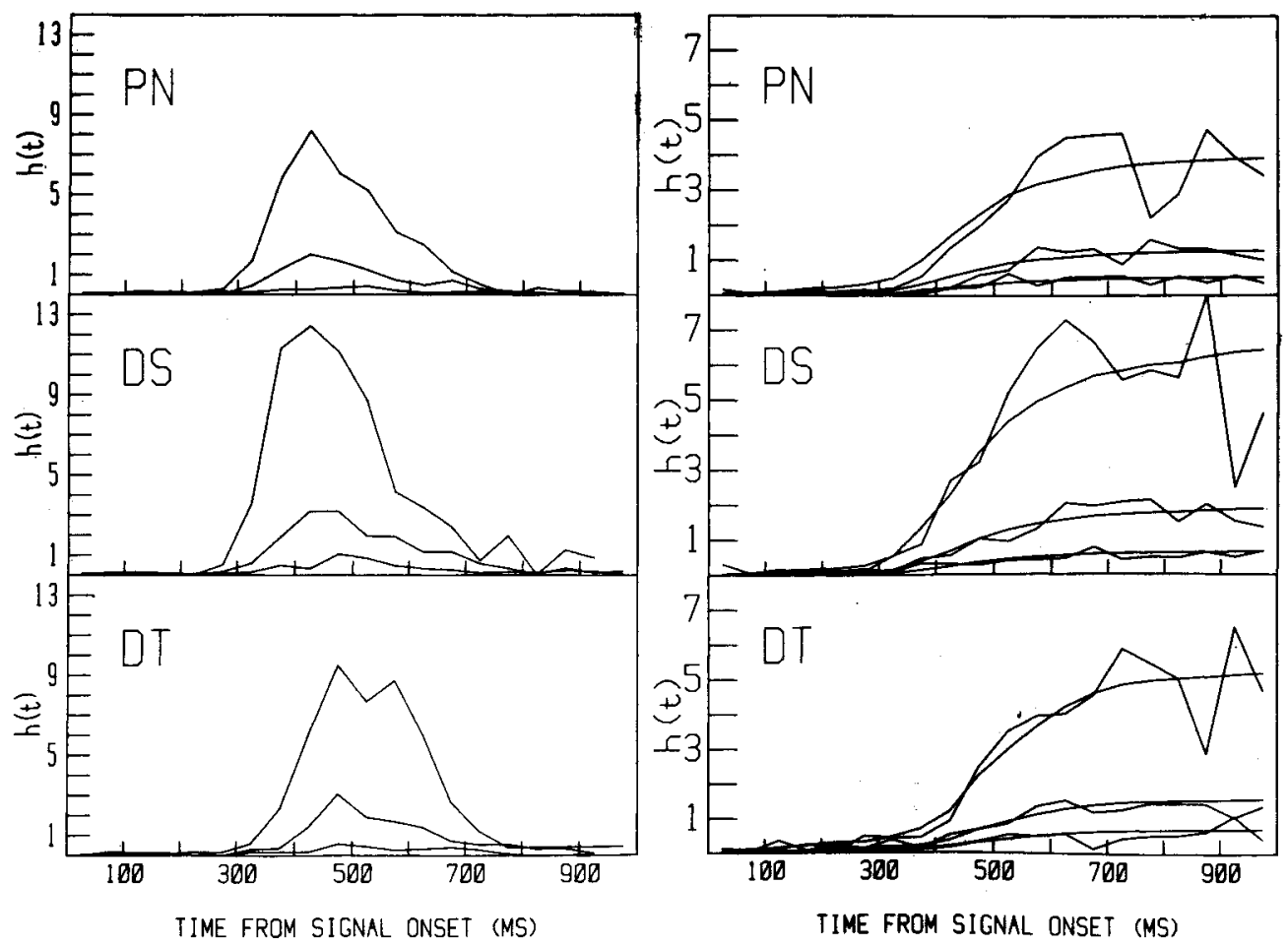

Figure 6. Hazard functions based on the reaction time data of Figure 5 . The left panel shows the data at the three signal levels for the 50-msec signal duration; the right panel shows similar data for the 1,000-msec signal duration. The smooth curve is a theoretical function and will be discassed later (see Figure 8). 
Figures 4, 5, and 6 show the basic distributions for all three observers. For the stimulus-wait and falsealarm distributions, backward cumulative probability graphs are provided in Figure 4 . The reactiontime density functions are presented in Figure 5 , and the hazard functions for all three signal levels are shown in Figure 6. The hazard functions are clearly different for the long and short signal durations. For the brief signal, the hazard functions rise to a peak at 400 to $500 \mathrm{msec}$. Note that the stronger signals produce the fastest rise. The hazard function diminishes and is nearly zero by $700 \mathrm{msec}$. Assuming 200-300 msec for the motor response, the short signal is likely to be heard $200-400 \mathrm{msec}$ after signal onset or not at all.

For the longer duration signal, the hazard functions of the response distributions are strikingly different. Again, they rise, beginning about $300 \mathrm{msec}$ after signal onset, and reach a plateau near $600 \mathrm{msec}$. The rate of rise depends on signal strength. The probability of detecting the signal, given that one has not already responded, then remains roughly constant.

Once more, there is no evidence of a change or transient detector. The hazard functions are completely consistent with an integration process having an accumulation window of $300-400 \mathrm{msec}$.

\section{DISCUSSION}

\section{Poisson Model with Two False-Alarm States}

We now will compare the behavior observed in this experiment with a simple Poisson model introduced earlier (Luce \& Green, 1970). This model is useful in understanding some of the first-order statistics and the probability of detection. The basic point of view is that, with no signal present, the observer can be likened to a Poisson process having a parameter $1 / \nu$ that is proportional to the probability of responding in the next small interval of time, $\Delta t$. The occurrence of a signal increases that probability. To a first approximation, a signal presentation causes an immediate increase in that probability that lasts while the signal is present. The response process is stochastically independent of the detection process and occurs with a latency of between 200 and $300 \mathrm{msec}$. The response latency is assumed to have a fixed upper bound; hence, the probability of a response latency greater than some time $\mathrm{T}$ is zero.

The implications of these assumptions have been derived earlier (Luce \& Green, 1970, Appendix 4), so we will simply summarize the major results here. At the beginning of a trial, we have a race between two Poisson processes: One, having parameter $1 / \lambda$, will initiate a signal; the other, having parameter $1 / v$, will initiate a false alarm. Because of this race, the signal-wait and false-alarm distributions are both exponentially distributed with parameter $1 /(v+\lambda)$, for times greater than the upper bound $T$ on the re- sponse latency. We assume this upper bound is no greater than 1 sec. A maximum likelihood estimate of the exponential parameter is simply the reciprocal of the mean of all events (either signal onset or false alarm) that exceed the upper bound. Thus, the reciprocals of the means of the tails of the signal-wait and false-alarm distributions, presented in Tables 1 and 2 , are estimates of $1 /(\nu+\lambda)$.

Since the means of the false-alarm and signal-wait distributions are seldom the same and often considerably different, we know immediately that some modification of this simple model is necessary. We propose the following: Assume the observer is in one of two possible states. State 1 is as we have describedthe observer initiates a false alarm with Poisson parameter $1 / \nu$. Assume state 1 occurs with probability p. We assume that a second state occurs with probability $1-p$, in which the probability of initiating a false alarm is virtually zero. In this second state, the observer is unwilling to make a false alarm; either the signal is clearly heard and a response initiated or the trial terminates with a miss. These assumptions require that we modify the original description, since two types of races may occur. If the observer is in state 1, our original description is correct; if the observer is in state 2 , the signal onset inevitably occurs and the exponential parameter of that distribution is simply $1 / \lambda$. The effects on the two distributions are different. The false-alarm distribution is still exponential with parameter $1 /(\nu+\lambda)$, since a false alarm can occur only if the observer is in state 1 . The signalwait distribution is a mixture of two exponentials. One occurs with probability $\mathbf{p}$ and has an exponential tail with parameter $1 /(\lambda+v)$. The other occurs with probability $1-p$ and has $1 / \lambda$ as the parameter of the tail.

The probabilities of the various events and the mean waits are as follows:

$$
\begin{gathered}
P(\text { false alarm })=P(F A)=p \frac{v}{v+\lambda} \\
P(\text { signal wait })=P(S W)=1-p+p\left(\frac{\lambda}{v+\lambda}\right) \\
\text { Mean(false alarm })=M(F A)=\frac{1}{v+\lambda} \\
\text { Mean(signal wait) }=M(S W)=(1-p) \frac{1}{\lambda}+p \frac{1}{v+\lambda}
\end{gathered}
$$

This last equation has some interesting implications if we substitute for $p$ in terms of the false-alarm probability from Equation 1 into Equation 4.

$$
\mathrm{M}(\mathrm{SW})=\left[1-\frac{v+\lambda}{v} \mathrm{P}(\mathrm{FA})\right] \frac{1}{\lambda}+\frac{v+\lambda}{v} \mathrm{P}(\mathrm{FA}) \frac{1}{v+\lambda}
$$




$$
\begin{aligned}
& =\frac{1}{\lambda}\left[1-\frac{v+\lambda}{\nu} \mathrm{P}(\mathrm{FA})+\frac{\lambda}{\nu} \mathrm{P}(\mathrm{FA})\right] \\
& =\frac{1}{\lambda}[1-\mathrm{P}(\mathrm{FA})] .
\end{aligned}
$$

Thus, the mean signal wait should equal the product of the complement of the false-alarm probability and the mean wait set by the experimenter, $1 / \lambda$. Another result of

$$
\mathrm{p}=\frac{\nu+\lambda}{\nu} \mathrm{P}(\mathrm{FA})
$$

together with the value of $v$ from Equation 3, is

$$
p=\frac{P(F A)}{1-\lambda M(F A)} ;
$$

thus for $p \leqslant 1$,

$$
1-\lambda \mathrm{M}(\mathrm{FA}) \geqslant \mathrm{P}(\mathrm{FA}) .
$$

In the 18 cases in which this inequality can be calculated, it is violated four times. All four violations occur in the first experiment, in which the number of observations is relatively small. The largest violation is $4.4 \%$, which is about 1.4 standard deviations, assuming binomial variability. The rest of the violations are $2 \%, 0.5 \%$, and $0.8 \%$. The bulk of the experimental data obey the inequality.

Next, we use the relation of Equation 5 to see how well we can predict the mean signal wait from the probability of a false alarm. Figure 7 shows the obtained and predicted signal waits. The six solid points represent the results of the second experiment, and are based upon considerably greater numbers of observations than are the open symbols based on the results of the first experiment.

To evaluate this prediction, we use a chi-square test. Let $y=M(S W)$ and $x=[1-P(F A)]$ and $\sigma_{y}$ and $\sigma_{x}$ be the respective standard deviations of these quantities. For each condition, we have an observed pair of values $\left(y_{i}, x_{i}\right)$ and the predicted relation $\hat{y}=(1 / \lambda) \hat{x}$. Thus,

$$
\left(\frac{y_{i}-\frac{1}{\lambda} \hat{x}}{\sigma_{y}}\right)^{2} \text { and }\left(\frac{x_{i}-\hat{x}}{\sigma_{x}}\right)^{2}
$$

are both chi-square distributed, since each quantity within parentheses is a standard normal deviate, with mean zero and unit variance. The value $\hat{x}$ is chosen to minimize the sum of the two squared quantities. Eighteen such calculations can be made, one for each

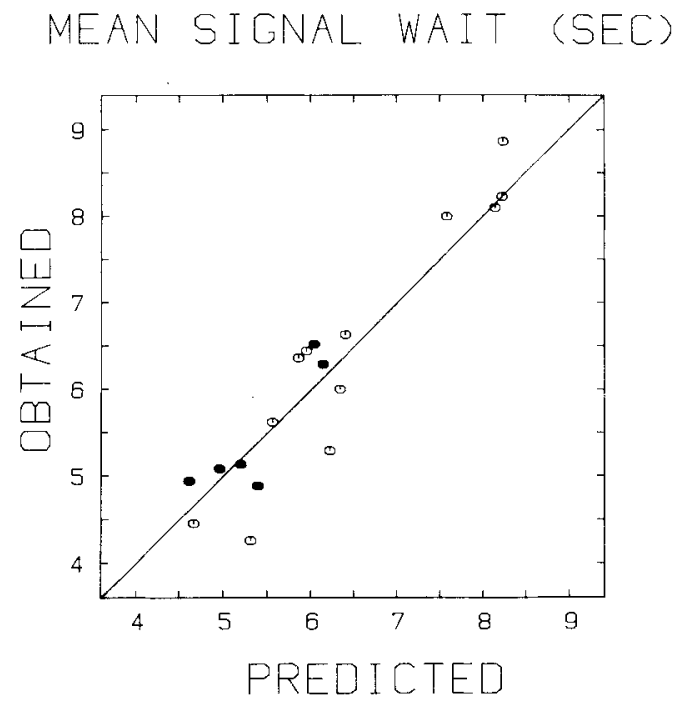

Figure 7. Predicted vs. obtained mean of signal-wait tail. The axes are logarithms of the obtained and predicted times. The prediction is based on Equation 5.

experimental condition. We use $\sqrt{\hat{\mathbf{x}}(1-\hat{\mathbf{x}}) / \mathbf{n}}$ as the estimate of $\sigma_{x}$. To estimate $\sigma_{y}$, we use the observed standard deviation for the signal-wait distribution divided by the square root of the number of observations, that is, the standard error of the signal wait.

The total chi-square for the 18 conditions was 50.4 with 18 degrees of freedom. Since the mean of a chisquare distribution is the degrees of freedom and the variance twice that number, the observed value of chi-square is several standard deviations above the mean and, hence, statistically significant. As is common with such tests, however, two conditions contribute about half of the total chi-square value. In spite of this failure, we believe the proposed model does remarkably well in accounting for the obvious discrepancy between the means of the signal-wait and false-alarm tails.

According to this view, only the false-alarm distribution is a single exponential distribution. Thu signal-wait distribution is, presumably, a mixture of two exponential distributions having parameters $1 / \lambda$ and $1 /(\nu+\lambda)$, and relative weights $1-p$ and $p$. One might expect the tails of the backward cumulative distribution to deviate from a straight line (see Figures 1 and 4). If the difference in exponential parameter is less than a factor of two, however, it is virtually impossible to observe any deviation from a straight line in the region where reasonable estimates of the probability can be obtained.

\section{Reaction-Time Hazard Function}

As mentioned previously, the form of the hazard function suggests a simple accumulation process. ${ }^{1}$ If this accumulation process is very simple, we may be able to predict the long-duration hazard function 
from the hazard function obtained with the shortduration signal. Suppose the contribution to the value of the hazard function at any instant is simply the convolution of the accumulation process and the Poisson parameter of the detection process. Without a signal, the Poisson parameter is some constant low value. We may model the onset of a brief signal as causing an instantaneous increase in this parameter for the duration of the signal. To this change in the input, the output of the accumulation process will slowly increase in value while the signal is on, and then decline when the signal is terminated. Given this view, the brief-signal hazard function should contain the information needed to predict the hazard function for the longer duration signal. Essentially, what we do is to take the $50-\mathrm{msec}$ signal hazard function and shift it in steps of $50 \mathrm{msec}$ for as long as the longer signal lasts, and then to add together all the shifted hazard functions.

That is,

$$
\alpha\left(I_{s}\right) h_{1000}(t)=\sum_{i=1}^{20} \alpha\left(I_{s}\right) h_{50}(t-i 50 \mathrm{msec}),
$$

where $h_{1000}(t)$ and $h_{50}(t)$ are the hazard functions for the $1,000-\mathrm{msec}$ and 50 -msec signals, respectively, and $\alpha\left(I_{s}\right)$ depends on the intensity of the signal. Unfortunately, the intensities of the signals we used at the two durations are quite different. The energy levels of the signal re the noise power density were 15,12 , and $9 \mathrm{~dB}$ for the 50-msec signal, corresponding to an . intensity or power re noise density of 28,25 , and $22 \mathrm{~dB}$. For the $1,000-\mathrm{msec}$ signal, the power ratio and energy ratio are equivalent, namely, 20, 17, and $14 \mathrm{~dB}$. Thus, the weakest signal intensity for the 50 -msec signal ( $22 \mathrm{~dB}$ ) is $2 \mathrm{~dB}$ higher than the strongest intensity $(20 \mathrm{~dB})$ for the 1,000 -msec signal. Somehow we must try to scale our brief-duration hazard function in our attempt to construct the long-duration hazard function.

Again, in this simple view, the intensity of the signal determines the size of the hazard function, but its form depends only on the accumulation process. We can test this idea to some extent by normalizing the short-duration hazard functions so that they all have the same total area. If signal intensity affects only the size and not the form of the hazard function, the normalized hazard functions should have similar shapes. For each of the three observers, the normalized functions are shown in Figure 8 and appear to have similar shapes.

Next, these three normalized functions were averaged to obtain a normalized hazard function for each observer; this function was shifted by 50 -msec steps along the time line, and the shifted functions were summed. The resultants, scaled to have the same total areas as the long-duration hazard functions, are

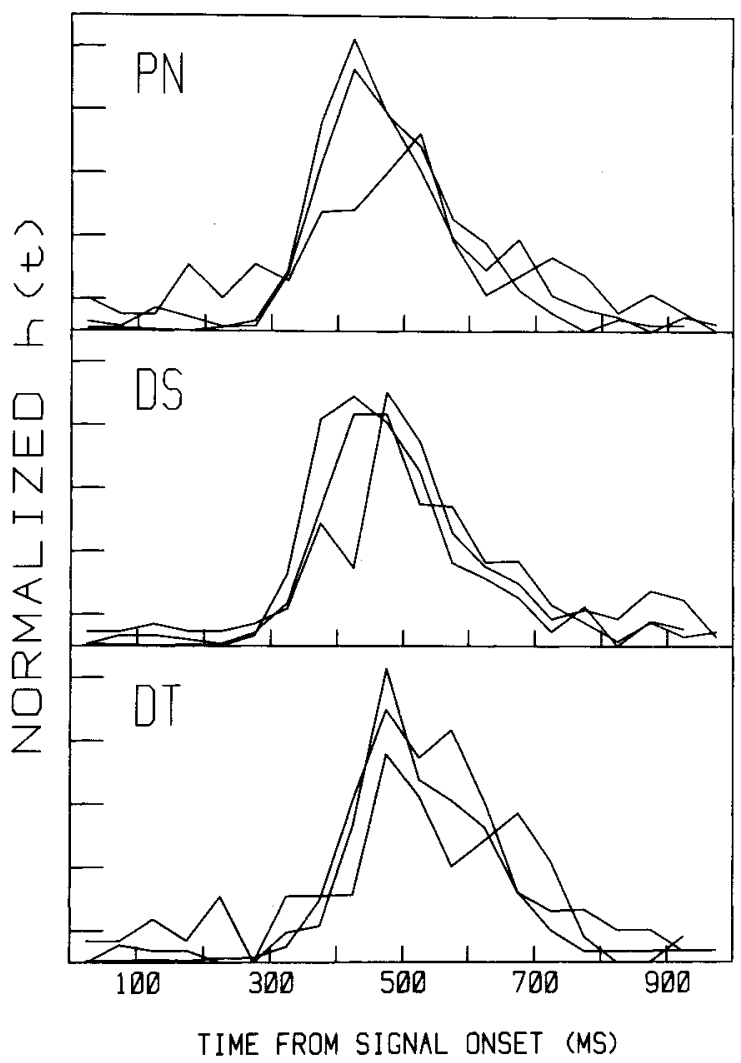

Figure 8. Normalized hazard functions for the three signal levels for the 50-msec signal duration. For each observer, the three hazard functions from the left panel of Figure 6 are equated for area, and the results are displayed. The normalized functions for each observer were subsequently avernged, shifted by S0-msec steps, and summed to generate the smooth lines shown in the right panel of Figure 6. For each empirical function, the height of the average normalized 50-msec function is adjusted to equate the areas of the observed and constructed long-duration functions.

shown as the smooth lines in the right panel of Figure 6. The resemblance to the actual data is striking. One free parameter is used in this exercise: The area of the fitted curve is set equal to that of the observed curve. Again, this procedure assumes that the intensity of the signal simply scales the size of the hazard function but does not affect its form. We postpone a statistical evaluation of these fits and next consider this scaling constant in more detail.

The area under the hazard function up to some time $t$ bears an intimate relation to the cumulative probability of detection up to that time. If $H(t)$ is the cumulative of the hazard function and $F(t)$ is the cumulative of the density function, then it is well known that

$$
H(t)=\ln \frac{1}{1-F(t)}
$$

Thus, for very small $F(t), H(t)$ is near zero, and as 
$F(t)$ approaches one, $H(t)$ becomes very large. Figure 9 plots $H(t)$ with $t=1 \mathrm{sec}$ as a function of the power of the signal used in the experiment. For each observer, the relation is nearly a power function. Table 3 summarizes the parameters of these fits for the three observers at both durations.

The effect of shifting and adding the 50-msec signal hazard function is, of course, to increase the cumulative hazard function $\mathrm{H}(1 \mathrm{sec})$ by about a factor of 10 , since the hazard functions peak at around $500 \mathrm{msec}$ and there are 10 steps of $50 \mathrm{msec}$ between 500 and $1,000 \mathrm{msec}$. We may check this approximation by computing the relative increase produced by shifting and adding the middle-level hazard function; the relative increases are $10.6,10.3$, and 8.8 for the three observers. Thus, if we use the weakest 50 -msec signal, the cumulative of the original hazard function is about 0.2 to 0.3 (see Figure 9), and by shifting and adding it together, the cumulative should be about 2 to 3 . This value is obviously still less than the cumulative value for the $1,000 \mathrm{msec}$ of most similar intensity, which is about 3 to 5 , for the $P / N_{0}=20 \mathrm{~dB}$ (see Figure 9). If we extrapolate either curve and compare

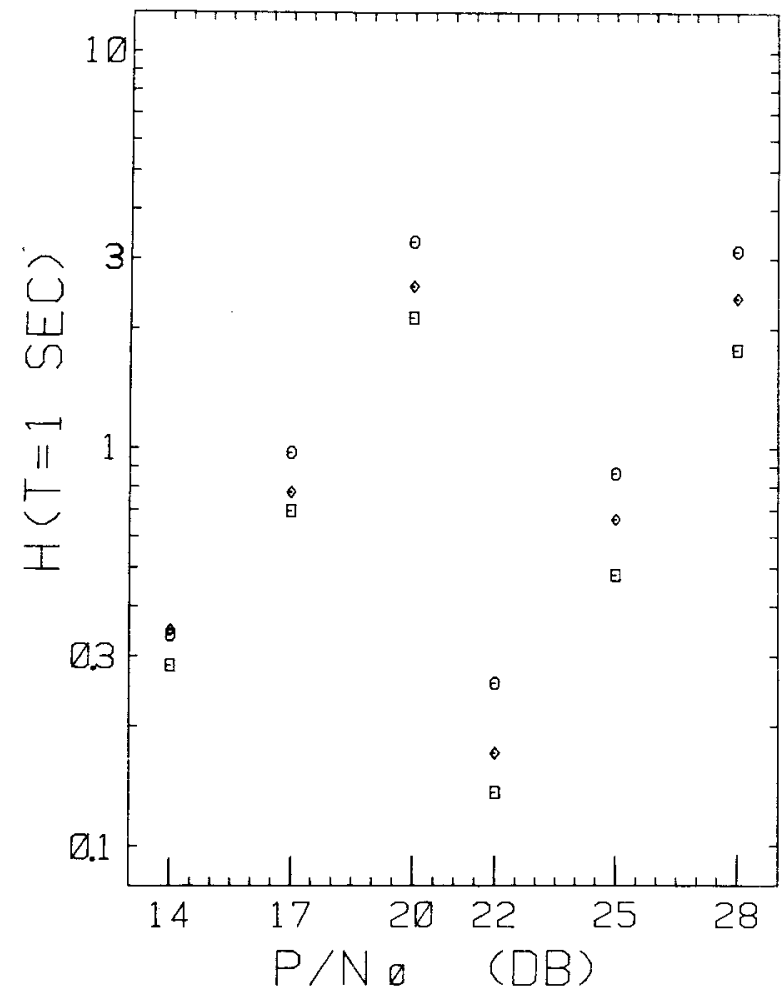

Figure 9. The cumulative hazard function of the reaction time distribution measured 1 sec after signal onset vs. the signal power, $P$, to noise power density $N_{0}$ in decibels. The signal power is the signal energy divided by the signal duration $\mathrm{E} / \mathrm{T}$. The points to the left represent the 1,000 -msec signal duration; the 50 -msec data are to the right. Since $10 \log (50 / 1000)=13 \mathrm{~dB}, \mathrm{a} P / \mathrm{N}_{0}$ of $25 \mathrm{~dB}$ corresponds to an $E / N_{0}$ of $12 \mathrm{~dB}$. The different points correspond to different observers: $\odot$ is D.S., $\odot$ is D.T., and $\square$ is P.N. The parameters describing this relation are tabulated in Table 3.
Table 3

Values of $k$ and $\alpha$ in Fits to Data of Figure 9

\begin{tabular}{ccccc}
\hline & \multicolumn{4}{c}{ Observers } \\
\cline { 2 - 5 } & PN & DS & DT & AVE \\
\hline \multicolumn{5}{c}{$\mathrm{T}=50 \mathrm{Msec}$} \\
$\mathrm{k}$ & 1.86 & 1.81 & 1.91 & 1.86 \\
$\mathrm{r}$ & $1.09^{*}$ & $2.57^{*}$ & $1.09^{*}$ & \\
& .9999 & .9999 & .9997 & \\
$\alpha$ & \multicolumn{5}{c}{$\mathrm{T}=1,000 \mathrm{Msec}$} \\
$\mathrm{k}$ & 1.45 & 1.65 & 1.43 & 1.51 \\
$\mathrm{r}$ & $2.54 \dagger$ & $1.64 \dagger$ & $3.20 \dagger$ & \\
\hline
\end{tabular}

Note-H(t=l sec $)=k\left(P / N_{0}\right)^{\alpha}$, where $P$ is power of signal, assuming a nominal l-ohm impedance. $N_{0}$ is noise power density. $P T=$ signal energy, where $T$ is signal duration.

$* \times 10^{-5}++\times 10^{-3}$.

the two at the same intensities, the discrepancy becomes even worse.

Evaluating the exact difference between the two regression lines for the 50- and 1,000-msec data is difficult, because they are not exactly parallel (see Table 3). The average slope for the 50 -msec data is 1.86 , and is only 1.51 for the longer duration data. But, using the average parameters, they differ by a factor of 27 at $P / N_{0}$ of $22 \mathrm{~dB}$ and by a factor of 31.2 at $\mathrm{P} / \mathrm{N}_{0}$ of $20 \mathrm{~dB}$, the signal powers for which they are most nearly equal. Thus, we are off by about a factor of 3 from the factor of 10 that we expect. Another difficulty with this comparison is that the false-alarm probabilities are not exactly equal for the 50- and 1,000 -msec conditions. But they do not differ by very much, and D.S.'s difference is opposite in sign to those of the other two observers. The most perplexing aspect of this discrepancy is the direction. If we believe the short-duration data are correct in all respects; then the longer signal shows more accumulation than can be obtained by adding the hazard functions. It is fairly easy to think of ad hoc reasons for the reverse to be true. Incomplete or imperfect accumulation would become prime candidates. The direction of this discrepancy is very puzzling, and certainly warrants further test of this procedure using signals of the same amplitude and different durations mixed within the same blocks to equate the falsealarm probability. We are unable to suggest any reasonable hypothesis to explain this discrepancy.

We conclude with a statistical evaluation of our attempt to predict the long-duration hazard function from adding and scaling the normalized short-duration hazard function. Once the hazard function is known, we can use Equation 7 to obtain the cumulative distributions of reaction times for each time, $t$, and hence, the density of reaction times, $f(t)$. We can compare the predicted and obtained number of responses for each $50-$ msec interval. The resulting chisquare values for the different observers are 99 for Observer P.N. (43 df), 77 for Observer D.S. (43 df), 
and 62 for Observer D.T. (37 df). The different degrees of freedom arise because different numbers of cells were combined to achieve an expected frequency of at least five responses. Obviously, all of these chisquare values are highly significant, but, given the sensitivity of this statistic, we are encouraged by values that are only about twice the degrees of freedom. There is little variation of the chi-square values as a function of level when we average over the three observers.

\section{SUMMARY}

Signal intensity and duration were studied using a signal whose onset followed an exponential wait. Two experiments were conducted: In the first, signal intensity was varied over blocks; in the second, to hold the response criterion fixed for all intensities, the signal level was selected at random on each trial. For both experiments, the signal-wait and false-alarm distributions are exponential in form. The mean of the tails of these distributions can be reasonably well predicted from a two-state false-alarm model. The shape of the hazard functions of the response distributions for the short-duration signal and the longduration signal are markedly different. The value of the cumulative hazard function, which is monotonically related to the probability of detection, is a power function of signal power. The short-duration hazard function can be used to predict, with good accuracy, the long-duration hazard function assuming a simple accumulation process and one free parameter. Unfortunately, the value of this free parameter is different from what we expect by about a factor of three.

\section{REFERENCE NOTE}

1. Burbeck, S. L., \& Luce, R. D. Evidence from auditory simple reaction time for both change and level detectors. Unpublished manuscript, 1981.

\section{REFERENCES}

GreEn, D. M., \& LuCE, R. D. Detection of auditory signals presented at random times. Perception \& Psychophysics, 1967, 2, 441-450.

Green, D. M., \& Luce, R. D. Detection of auditory signals presented at random times, III. Perception \& Psychophysics, $1971,9,257-268$.

LuCE, R. D., \& Green, D. M. Detection of auditory signals presented at random times, II. Perception \& Psychophysics, $1970,7,1-14$.

\section{NOTE}

1. We are aware that Burbeck and Luce (Note 1) have analyzed auditory detection data and found evidence for both change and level detectors. There are several experimental differences between the two studies. Among the more salient is that they studied the detection of the termination of the signal. We are unable to offer any reasons for the different results.

(Manuscript received September 8, 1981; accepted for publication September 29, 1981.) 